\title{
Epidermal growth factor improves the migration and contractility of aged fibroblasts cultured on 3D collagen matrices
}

\author{
DAEHWAN KIM $^{1 *}$, SO YOUNG KIM ${ }^{2,3 *}$, SEOG KYUN MUN ${ }^{4}$, SANGMYUNG RHEE ${ }^{1}$ and BEOM JOON KIM ${ }^{2,3}$ \\ ${ }^{1}$ Department of Life Science, College of Natural Sciences, ${ }^{2}$ Major in Biomedical Science, \\ Department of Medicine, Graduate School, Chung-Ang University; Departments of ${ }^{3}$ Dermatology and \\ ${ }^{4}$ Otolaryngology-Head and Neck Surgery, Chung-Ang University College of Medicine, Seoul, Republic of Korea
}

Received September 3, 2014; Accepted January 12, 2015

DOI: $10.3892 /$ ijmm.2015.2088

\begin{abstract}
Epidermal growth factor (EGF) plays a critical role in fibroblasts by stimulating the production of collagen and supports cell renewal through the interaction between keratinocytes and fibroblasts. It is well known that the contractile activity of fibroblasts is required for the remodeling of the extracellular matrix (ECM), which contributes to skin elasticity. However, the role of EGF in the contraction of aged fibroblasts under 3-dimensional (3D) culture conditions is not yet fully understood. In the present study, we demonstrated that young fibroblasts spread and proliferated more rapidly than aged fibroblasts under 2-dimensional (2D) culture conditions. Cell migration assay using a nested collagen matrix revealed that the migration of young fibroblasts was also greater than that of aged fibroblasts under $3 \mathrm{D}$ culture conditions. However, the addition of recombinant human EGF (rhEGF) resulted in the enhanced migration of aged fibroblasts; the migration rate was similar to that of the young fibroblasts. The aged fibroblasts showed decreased cluster formation compared with the young fibroblasts on the collagen matrix, which was improved by the addition of rhEGF. Furthermore, cell contraction assay revealed that the basal contractility of the aged fibroblasts was lower than that of the young fibroblasts; however, following treatment with rhEGF, the contractility was restored to levels similar or even higher to those of the young fibroblasts. Taken together, our results suggest that rhEGF is a potential renewal agent that acts to improve the migration and contraction
\end{abstract}

Correspondence to: Professor Beom Joon Kim, Department of Dermatology, Chung-Ang University College of Medicine, 224-1 Heukseok-dong, Dongjak-gu, Seoul 156-755, Republic of Korea E-mail: beomjoon@unitel.co.kr

Professor Sangmyung Rhee, Department of Life Science, College of Natural Sciences, Chung-Ang University, 84 Heukseok-ro, Dongjak-gu, Seoul 156-756, Republic of Korea

E-mail: sangmyung.rhee@cau.ac.kr

*Contributed equally

Key words: epidermal growth factor, fibroblasts, cell migration, cell contraction, platelet-derived growth factor, lysophosphatidic acid, three-dimensional collagen matrix of aged fibroblasts more efficiently than young fibroblasts under $3 \mathrm{D}$ culture conditions; thus, EGF may have valuable regenerative effects on aged skin.

\section{Introduction}

Skin aging is a biological process that involves cutaneous aging and photo-aging, both of which are associated with harmful environmental insults, including UV radiation, heat, gravity and smoking (1). More specifically, cutaneous aging comprises the loss of elasticity, which causes wrinkling of the skin through the deprivation of extracellular matrix (ECM) $(2,3)$. Treatments for skin aging include herbal extracts, vitamins and growth factors, which have been established to promote ECM protein synthesis and to reduce degradation enzymes of the ECM, thereby delaying the progression of skin aging (4-6).

Several growth factors and cytokines are involved in the regulation of skin homeostasis and development (7), all of which are important for the preservation of cutaneous functions by acting as autocrine and paracrine factors (8). Furthermore, several recombinant cytokines and growth factors are currently used in clinical practice to treat patients with various diseases. These cytokines and growth factors control the regulatory functions of several powerful and complex signaling networks. Among these, the epidermal growth factor (EGF) and its receptor (EGFR) command a wide spectrum of diverse biological effects, including cellular proliferation, differentiation, wound healing, hair follicle morphogenesis and tumorigenesis.

EGF is a dominant protein that is produced by platelets, macrophages and monocytes, and stimulates the differentiation of progenitor cells within the epithelium and sebaceous glands, as well as fibroblasts during wound healing and hair follicle morphogenesis $(9,10)$. EGF binds to erythroblastic leukemia viral (v-erb-b) oncogene homolog 1 (ErbB1, EGFR and HER1), which is an important growth factor receptor that helps to control the rate of DNA synthesis, stimulate translation, and increase protein phosphorylation on the surfaces of cells, which in turn induces the further differentiation of skin cells (11-13). In the context of human skin, EGF has been identified as a useful agent for improving acne, aging and chronic skin ulcers for skin homeostasis $(6,14)$. From a histopathological perspective, EGFR is strongly expressed in the basal layer comprising epidermal keratinocytes and the 
outer root sheath (ORS) of hair follicles, and is involved in the regulation of proliferation and differentiation $(15,16)$. EGF has also been reported to help mitigate the effects of aging by supporting skin regeneration by stimulating cell renewal through the interaction of keratinocytes and fibroblasts $(17,18)$. In the dermis, EGF plays a critical role in fibroblasts by stimulating the production of collagen through the activation of EGFR. Whereas younger dermal fibroblasts have an adequate capacity for ECM remodeling, aged dermal fibroblasts are present in fewer numbers and exhibit a decreased capacity for collagen synthesis (19). Consistent with these data, aged dermal fibroblasts within the ECM exhibit reduced EGFR expression, which is associated with decreased cell migration and proliferation, and ultimately the loss of flexibility and elasticity, as well as poor mechanical tension $(20,21)$. Therefore, the contractile activity of fibroblasts is important for ECM remodeling, which contributes to skin elasticity (22).

The skin is composed of an epidermis, dermis and subcutaneous tissue and exhibits multiple functions (23). Due to this characteristic of skin, monolayer cell culture models cannot fully recapitulate the functional complexity of human skin. Thus, tissue-engineered skin substitutes utilizing alternative skin scaffold approaches, such as 3-dimensional (3D) collagen matrices may represent more effective systems for skin research $(24,25)$. In addition, tissue-engineered skin substitutes provide a novel platform for testing the effects and biological reactions of pharmacological agents on the assembly of tissuelike structures, and may be suitable for replacing some in vivo experiments and preliminary real human skin studies $(26,27)$. Measurements of floating collagen matrix contraction, stressedreleased matrix contraction and nested collagen matrices have been to increased the understanding growth factor function during physiological processes in fibroblasts $(28,29)$. Moreover, $3 \mathrm{D}$ collagen matrix systems used to study ECM have been shown to simulate a wide spectrum of diverse bioregulatory functions. Therefore, tissue-engineered skin substitutes may represent a useful system for the study of skin biological responses. Nevertheless, the effects of EGF on the contraction of aged human fibroblasts in artificial skin tissue, such as the 3D collagen matrix have not yet been fully determined.

In the present study, we investigated whether treatment with synthetic recombinant human EGF (rhEGF) improves the migration and contraction of aged human fibroblasts in under 3D collagen culture conditions. We demonstrated that treatment with rhEGF increased the migration of aged fibroblasts; the migration rate reached levels similar to those of young fibroblasts. We also demonstrated that treatment with rhEGF increased the contractility of aged fibroblasts to a greater extent than that of young fibroblasts in a 3D collagen matrix, indicating that rhEGF may be a potential renewal agent with valuable regenerative effects on aged skin.

\section{Materials and methods}

Materials. Dulbecco's modified Eagle's medium (DMEM) powder and fetal bovine serum (FBS) were purchased from Invitrogen (Gaithersburg, MD, USA). Alexa Fluor 488 phalloidin was obtained from Molecular Probes (Eugene, OR, USA). 4',6-Diamidino-2-phenylindole dihydrochloride (DAPI), 3-(4,5-dimethylthiazol-2-yl)-2,5-diphenyl tetrazolium bromide
(MTT) and lysophosphatidic acid (LPA) were purchased from Sigma-Aldrich (St. Louis, MO, USA). Fluoromount G was obtained from Southern Biotechnology Associates (Birmingham, AL, USA). Type I rat tail collagen (10.6 mg/ ml) was purchased from BD Biosciences (Bedford, MA, USA). Platelet-derived growth factor (PDGF) was obtained from Upstate Biotechnology (Lake Placid, NY, USA). Fatty acid-free bovine serum albumin (BSA) was obtained from Calbiochem (San Diego, CA, USA). Recombinant human epidermal growth factor (rhEGF) was provided by BIO-FD\&C Co., Ltd. (Hwasun, Korea).

Cell isolation and culture of fibroblasts. Primary human skin fibroblasts representing young and aged fibroblasts were obtained from patients aged 12 and 55 years, respectively. Dermal fibroblasts were obtained from human foreskins during circumcision or plastic surgery, after obtaining written informed consent according to ethical guidelines, from the Medical Ethics Committee of the Chung-Ang University Hospital (IRB14-0004). Skin specimens were processed according to the methods described in the study by Rheinwald and Green (30), which we modified by including thermolysin (Sigma-Aldrich). The young and aged fibroblasts were cultured in DMEM supplemented with $10 \%$ FBS and $1 \%$ penicillinstreptomycin (Welgene, Daegu, Korea) $(10,000 \mathrm{U} / \mathrm{ml}$ and $10,000 \mu \mathrm{g} / \mathrm{ml}$, respectively). Cell culture and experimental incubations were performed at $37^{\circ} \mathrm{C}$ in a $5 \% \mathrm{CO}_{2}$ incubator. Cultured dermal fibroblasts were harvested after 5-6 days by trypsinization and expanded to new T25 culture flasks (Nunc, Roskilde, Denmark). Serial passaging was performed by trypsinization when the subcultures reached $80-90 \%$ confluency. Fibroblasts obtained from 12 and 55 year-old patients were used at passage 4 for downstream experiments.

Morphological analysis and cell proliferation assay. Cell morphology was evaluated after $72 \mathrm{~h}$ by phase contrast microscopy. Photographs of the cells were acquired using an Olympus CKX41 inverted microscope with DP Controller software (Olympus Optical Co., Tokyo, Japan). Cell proliferation was determined by MTT assay. Briefly, the fibroblasts were seeded at a density of $1.5 \times 10^{4}$ cells/well into 24 -well plates and cultured for $18 \mathrm{~h}$, after which the cells were washed twice with PBS and cultured for an additional $72 \mathrm{~h}$ in serum-free medium. Subsequently, $500 \mu \mathrm{l}$ of MTT solution $(5 \mathrm{mg} / \mathrm{ml})$ in serum-free medium were added followed by incubation at $37^{\circ} \mathrm{C}$ for $4 \mathrm{~h}$. The supernatant was then removed, and the formazan crystals were dissolved in $200 \mu \mathrm{l}$ of dimethyl sulfoxide (DMSO). The plate was shaken at room temperature for $30 \mathrm{~min}$, and the optical density was measured at $540 \mathrm{~nm}$ using an ELISA reader ( VersaMax; Molecular Devices, Sunnyvale, CA, USA).

Senescence-associated (SA) $\beta$-galactosidase staining. For SA $\beta$-galactosidase staining, the cells were divided into 2 experiment groups as follows: i) young dermal fibroblasts; and ii) aged dermal fibroblasts. SA- $\beta$-gal activity, a molecular marker of dermal fibroblast cellular aging in vitro, was determined using a senescent cell staining kit (Cell Signaling Technology, Danvers, MA, USA) according to the manufacturer's instructions. Blue staining was visible after $4 \mathrm{~h}$ of incubation with a $\beta$-galactosidase staining solution containing 5-bromo-4chloro- 
A
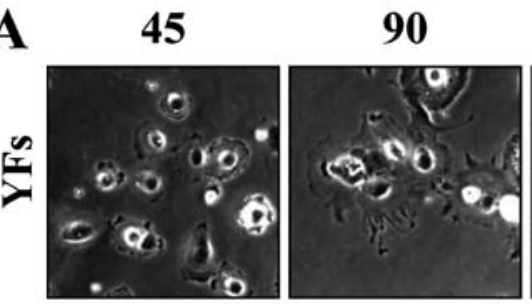

180 360 (min)
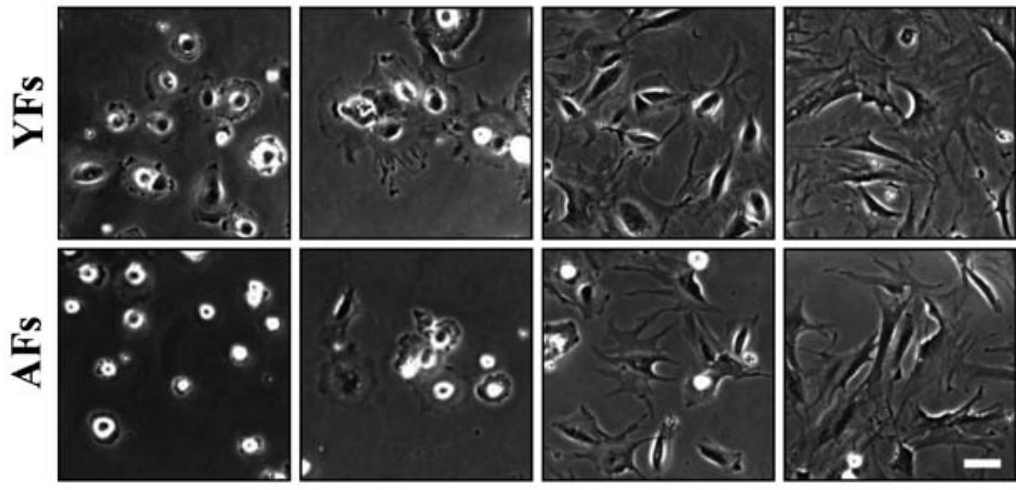

B

YFs
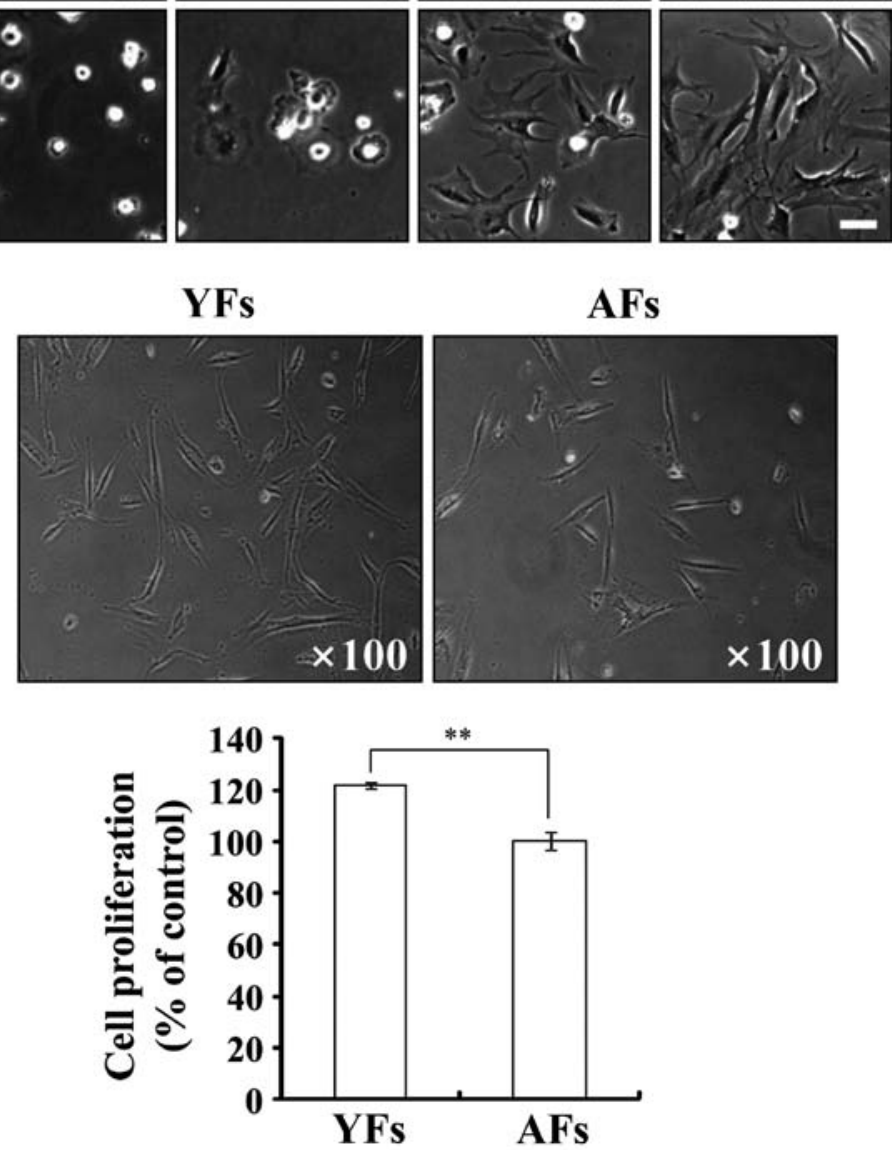

Figure 1. Characteristics of young and aged fibroblasts. (A) Time-dependent cell spreading of young fibroblasts (YFs) and aged fibroblasts (AFs). The cells were incubated for the indicated periods of time. Photomicrographs were acquired using a digital video camera, as described in Materials and methods. (B) Proliferation of young and old fibroblasts. Cells were incubated for $72 \mathrm{~h}$ in culture medium. After an additional incubation for $4 \mathrm{~h}$ with $1 \mathrm{mg} / \mathrm{ml} \mathrm{MTT}$ the amount of formazan was determined at $540 \mathrm{~nm}$ in an ELISA reader. Results are expressed as the percentage of control in 6 replicate cultures. ${ }^{* *} \mathrm{P}<0.01$, as indicated by Student's t-test. Data are presented as the means $\pm \mathrm{SD}, \mathrm{n}=6$.

3 -indolyl- $\beta$-D-galactosidase $\left(\mathrm{X}\right.$-gal) at $37^{\circ} \mathrm{C}$. The percentage of blue cells in 100 cells observed under a light microscope was calculated. Cell morphology after SA- $\beta$-galactosidase staining was observed as described above.

Cell contraction assay. For stressed-released matrix contraction (SMC), collagen matrices $(150 \mu 1,1 \mathrm{mg} / \mathrm{ml}$ collagen, $6.5 \times 10^{4}$ cells/matrix) were polymerized for $24 \mathrm{~h}$ in DMEM with $5 \mathrm{mg} / \mathrm{ml} \mathrm{BSA}, 10 \mu \mathrm{M}$ LPA or $1 \mu \mathrm{M}$ rhEGF, released from the culture surface, and then incubated for $2 \mathrm{~h}$ floating in the same medium. At the end of the incubation period, the matrices were fixed with $3.7 \%$ paraformaldehyde and the extent of matrix contraction was measured.

Immunofluorescence staining. Immunofluorescence staining was carried out as previously described (31). For actin staining, we used Alexa Fluor 488-conjugated phalloidin. To detect cell nuclei, the samples were stained with DAPI at $1 \mu \mathrm{g} / \mathrm{ml}$ for $1 \mathrm{~min}$. Images were collected using a fluorescence microscope (Eclipse 80i; Nikon, Tokyo, Japan) with Plan Fluor 10x/0.30, Plan Apo 20x/0.75 and Plan Fluor 40x/0.75 infinity-corrected objectives. Images were acquired using a digital camera (digital sight DS-Qi1Mc; Nikon) and NIS elements image analysis software (Nikon). Image processing was carried out using Photoshop 11.0 (Adobe). A phase contrast inverted microscope (Olympus Optical Co.) was used to visualize transparent objects such as cells.

Cell migration assay. To measure 3D cell migration using nested collagen matrices, floating collagen matrices $(1 \mathrm{mg} / \mathrm{ml})$ were incubated for $4 \mathrm{~h}$ in DMEM including $10 \% \mathrm{FBS}$, after which the cell-containing contracted matrices were re-embedded in $200 \mu \mathrm{l}$ of collagen $(1 \mathrm{mg} / \mathrm{ml})$ and incubated for an additional $24 \mathrm{~h}$ in DMEM with various growth factors including PDGF 

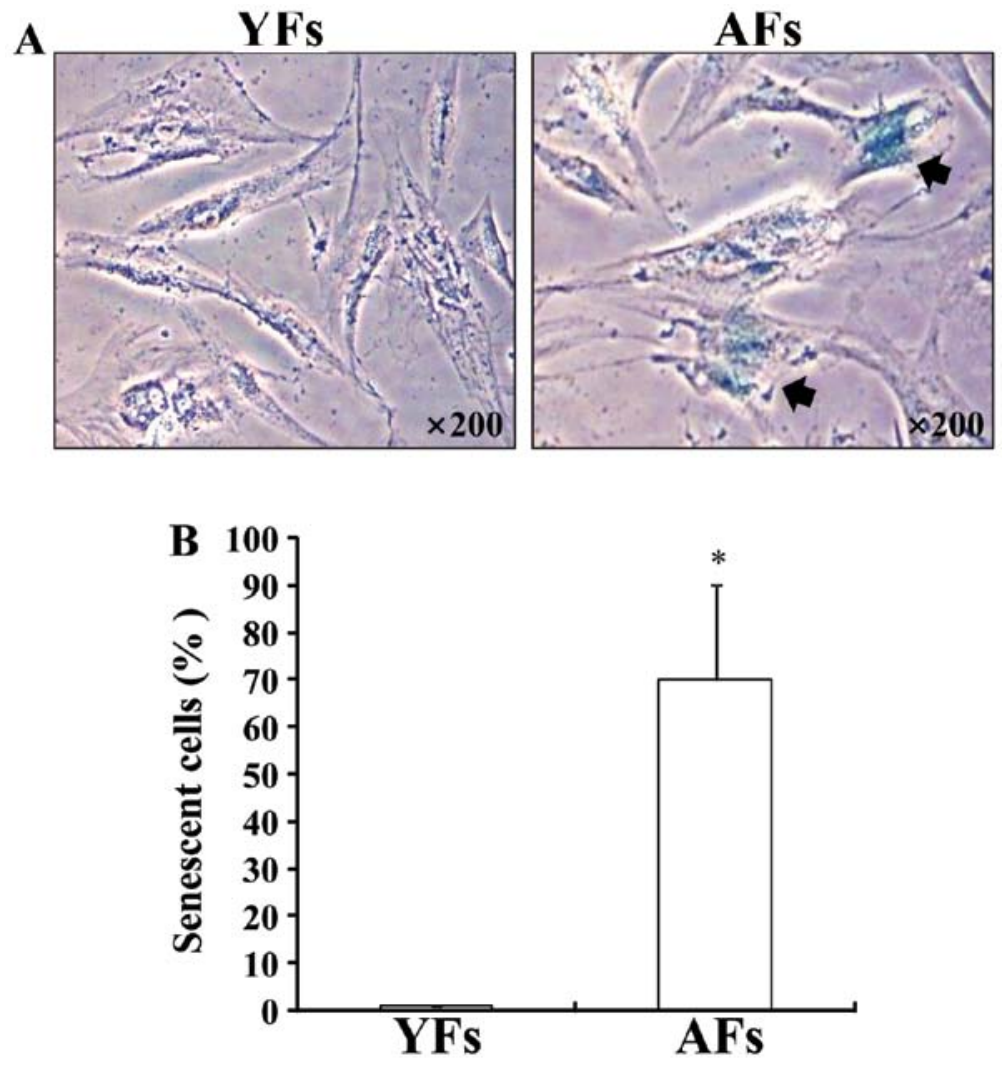

Figure 2. Senescence-associated (SA) $\beta$-galactosidase staining of fibroblasts. (A) SA $\beta$-galactosidase staining of young fibroblasts (YFs) and aged fibroblasts (AFs). Positive blue staining for SA $\beta$-galactosidase staining present in aged fibroblasts is indicated by arrows. After staining, the cells were imaged by phase contrast microscopy. Micrographs are shown at x200 magnification. (B) Quantitative analysis of positive $\beta$-galactosidase-stained cells in the 2 (young and aged) populations of fibroblasts. The percentage of cells positive for SA $\beta$-galactosidase staining was significantly increased in aged fibroblasts compared with young fibroblasts. Each measurement was made in triplicate. ${ }^{*} \mathrm{P}<0.05$ as indicated by Student's t-test. Data are presented as the means $\pm \mathrm{SD}, \mathrm{n}=3$.

$(50 \mathrm{ng} / \mathrm{ml})$ and EGF $(1 \mu \mathrm{M})$. At the end of the incubation period, the matrices were fixed and stained with phalloidin and DAPI to visualize actin and cell nuclei, respectively. The cell migration index was calculated by counting the average number of cells that had migrated out of dermal equivalents in 5 randomly selected microscopic fields at x10 magnification. To perform the 3D cell clumping assay, the cells were seeded on $1 \mathrm{mg} / \mathrm{ml}$ collagen matrices and incubated for $24 \mathrm{~h}$ in DMEM containing $5 \mathrm{mg} / \mathrm{ml} \mathrm{BSA,} 10 \mu \mathrm{M}$ LPA or $1 \mu \mathrm{M}$ rhEGF. At the end of the incubation period, the samples were fixed and stained with phalloidin and DAPI as described above.

Statistical analysis. Results are expressed as the means \pm SD. Data were analyzed using the Student's t-test and P-values $<0.05$ were considered to indicate statistically significant differences.

\section{Results}

Characterization of young and aged fibroblasts under $2 D$ culture conditions. To characterize the differences between the young and aged fibroblasts, we first compared cell spreading, proliferation and the rate of senescence. As shown in Fig. 1A, the young fibroblasts initially spread more rapidly than the aged fibroblasts at an early time point, although after $3 \mathrm{~h}$ this difference was decreased and by $6 \mathrm{~h}$ both groups of cells had spread completely. In addition, as shown in Fig. 1B, the young fibroblasts proliferated more rapidly than the aged fibroblasts. We then observed positive blue SA- $\beta$-galactosidase staining in the aged fibroblasts, which suggested the presence of senescent cells (Fig. 2A). Quantitative analysis revealed that the percentage of SA- $\beta$-gal-positive cells was higher $(\mathrm{P}<0.05)$ in the aged fibroblasts compared to the young fibroblasts (Fig. 2B). To analyze the effects of rhEGF on the proliferation of young and aged fibroblasts, we performed MTT assay with both cells treated with rhEGF. rhEGF promoted the proliferation of the young fibroblasts, but not the aged fibroblasts by $32 \pm 0.69 \%$ at a concentration of $1 \mu \mathrm{M}$ (Fig. 3). Taken together, these results suggest that young fibroblasts have an increased capacity for cellular proliferation compared with aged fibroblasts.

Migration of young and aged fibroblasts under $3 D$ culture conditions. We used a nested collagen matrix to measure fibroblast migration under 3D culture conditions. PDGF has previously been shown to promote fibroblast migration in a nested collagen matrix (32), and thus PDGF-treated cells were used as a positive control. As shown in Fig. 4, the migration rate of the young fibroblasts was greater than that of the aged fibroblasts under growth factor-free conditions. However, treatment with PDGF enhanced the migration of the aged fibroblasts to a level similar to that of the young fibroblasts. Similarly, treatment with rhEGF also increased the migration rate of the aged fibroblasts $(18 \pm 0.7)$ to a greater extent than that of the young fibroblasts $(13 \pm 4.5)$, even though the total migration seemed higher in the young 


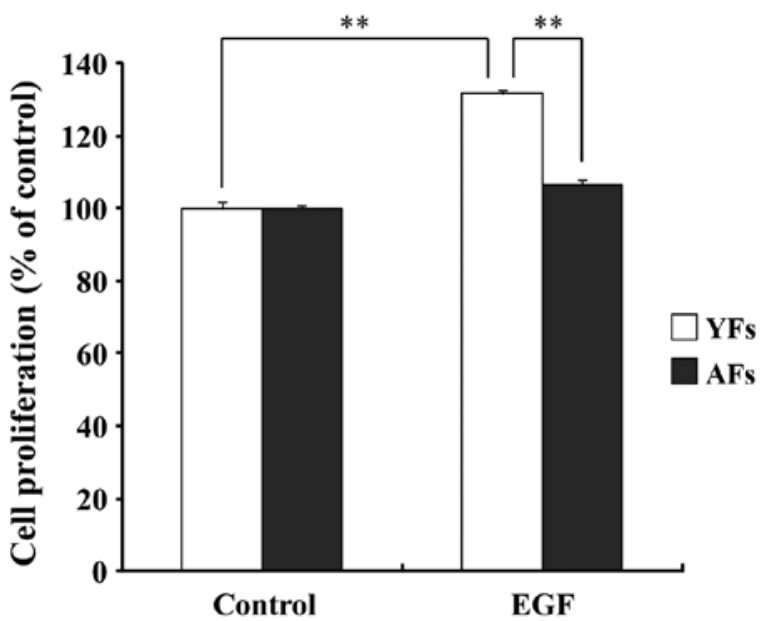

Figure 3. Effect of recombinant human epidermal growth factor (rhEGF) on the proliferation of young fibroblasts (YFs) and aged fibroblasts (AFs). The cells were treated for $72 \mathrm{~h}$ with $1 \mu \mathrm{M}$ rhEGF. After an additional incubation for $4 \mathrm{~h}$ with $5 \mathrm{mg} / \mathrm{ml} \mathrm{MTT}$, the formation of formazan was determined at $540 \mathrm{~nm}$ in an ELISA reader. Each measurement was made in triplicate, and data represent the means $\pm \mathrm{SD} .{ }^{* *} \mathrm{P}<0.001$ as indicated by the Student's t-test.

fibroblasts, indicating that EGF has a promigratory effect and that it enhances the migration of aged fibroblasts more effectively than that of young fibroblasts in a 3D collagen matrix.

Contraction of young and aged fibroblasts under $3 D$ culture conditions. Fibroblasts cultured in a 3D collagen matrix are known to exhibit dendritic extension morphology. Specifically,
PDGF promotes dendritic extension, whereas LPA or serum stimulates fibroblast contraction (31). As shown in Fig. 5A, the young fibroblasts had fewer dendritic extensions and protruded less into the matrix than the aged fibroblasts under basal (BSA) and LPA culture conditions, particularly at an early time point $(4 \mathrm{~h})$. These results suggest that young fibroblasts have an increased contractility compared with aged fibroblasts. In addition, as shown in Fig. 5B, the young fibroblasts migrated inward and generated small cell clusters even under basal culture conditions, whereas the same effect was not observed for the aged fibroblasts. However, both the young and aged fibroblasts formed large clusters when stimulated with either LPA or rhEGF, indicating that both agents were able to promote the contraction of fibroblasts. Based on these results, we hypothesized that the basal contractile activity of young fibroblasts may be higher than that of aged fibroblasts. As shown in Fig. 5B, the sizes of the clusters formed by the young and aged fibroblasts did not differ significantly following culture with rhEGF and LPA, which suggests that EGF promotes the contractile activity of aged fibroblasts more effectively than that of young fibroblasts as does treatment with LPA. To evaluate this possibility, we performed a SMC assay with young and aged fibroblasts. Unlike floating matrix contraction (FMC), PDGF does not induce contraction and only LPA or serum promote contraction in the SMC (33). As shown in Fig. 6, the contractility of the young fibroblasts was greater than that of the aged fibroblasts under basal conditions, while the contractile activity of the aged fibroblasts was increased to a level similar to that of the young fibroblasts in the presence of LPA. Of note, rhEGF promoted the contractility of the aged fibroblasts to levels similar or even higher to
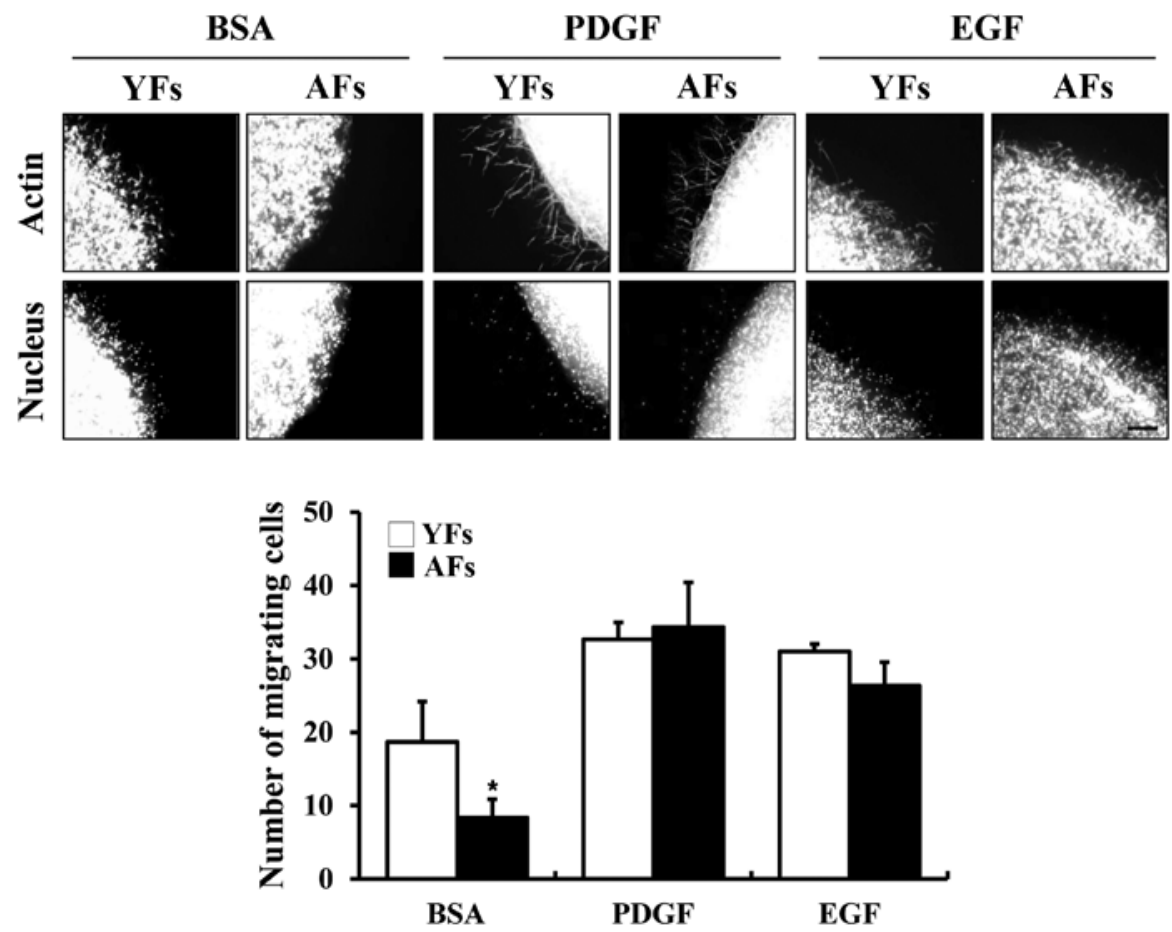

Figure 4. Migration of young fibroblasts (YFs) and aged fibroblasts (AFs) under 3D culture conditions. Nested collagen matrices were incubated for $24 \mathrm{~h}$ in DMEM in the presence of $5 \mathrm{mg} / \mathrm{ml} \mathrm{BSA,} 50 \mathrm{ng} / \mathrm{ml}$ platelet-derived growth factor (PDGF) or $1 \mu \mathrm{M}$ recombinant human epidermal growth factor (rhEGF). Migrating fibroblasts were visualized by staining for actin and nuclei. Cells that migrated from the border of the matrix in 6 fields were counted and the average number of migrating cells is shown. Scale bar, $200 \mu \mathrm{m}$. ${ }^{*} \mathrm{P}<0.05$ as indicated by the Student's t-test. 
A $4 \mathrm{~h}$

$24 \mathrm{~h}$
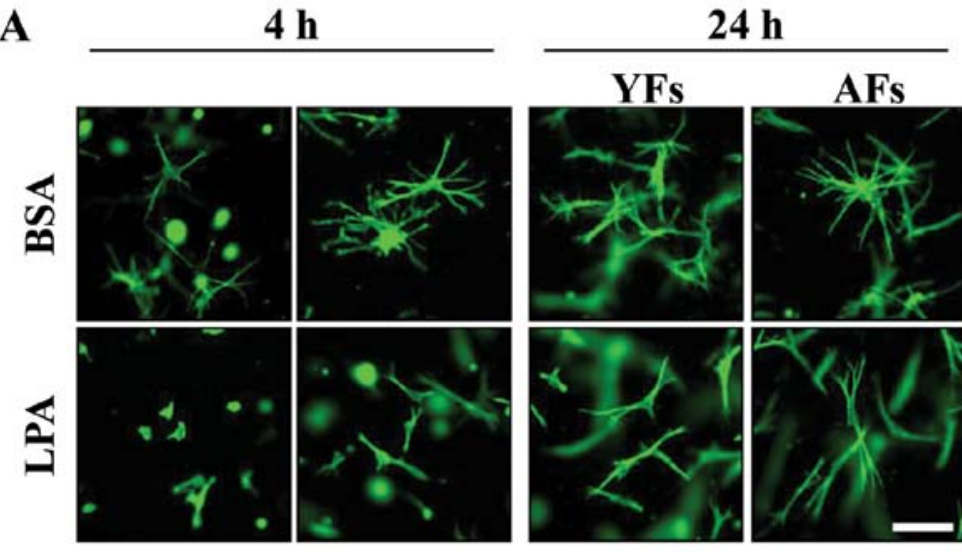

B

BSA

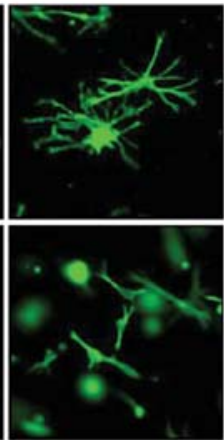

LPA
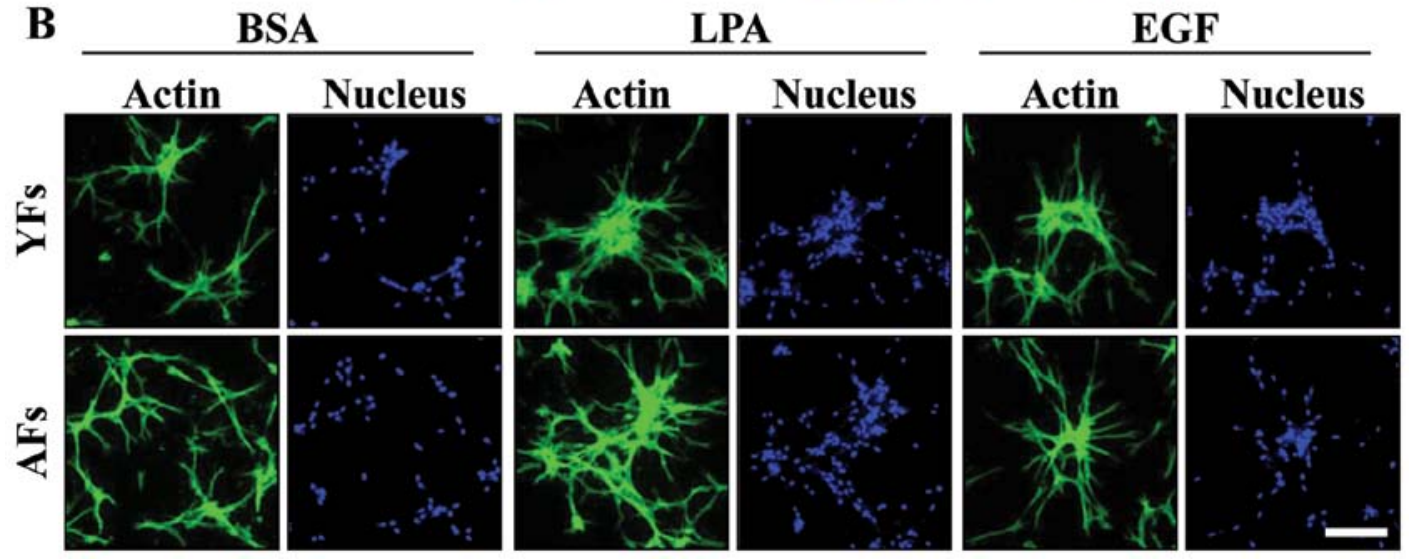

Figure 5. Morphology and cluster-forming ability of young fibroblasts (YFs) and aged fibroblasts (AFs) under in 3D culture conditions. (A) Fibroblasts were incubated in a 3D collagen matrix $(1 \mathrm{mg} / \mathrm{ml})$ and incubated for the indicated periods of time. After incubation, the cells were fixed and stained with phalloidin. Scale bar, $200 \mu \mathrm{m}$. (B) Fibroblasts were seeded on the top of the collagen matrix and incubated in DMEM with $5 \mathrm{mg} / \mathrm{ml}$ bovine serum albumin (BSA), $1 \mu \mathrm{M}$ recombinant human epidermal growth factor (rhEGF), or $10 \mu \mathrm{M}$ lysophosphatidic acid (LPA) for $24 \mathrm{~h}$. At the end of the incubation period, the cells were fixed and stained with phalloidin and DAPI. Scale bar, $200 \mu \mathrm{m}$.
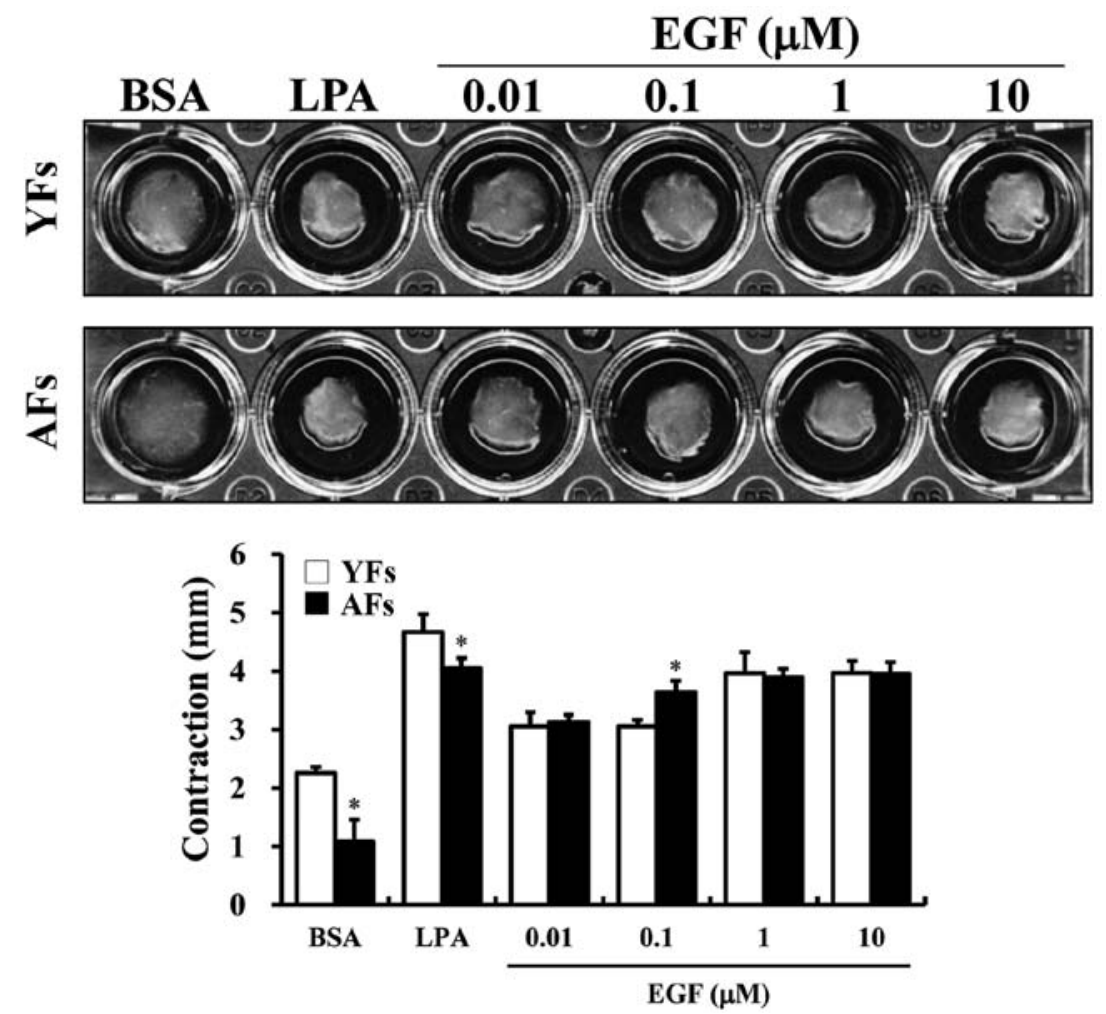

Figure 6. Contraction of young fibroblasts (YFs) and aged fibroblasts (AFs). Fibroblasts were incubated in stressed matrix with 5 mg/ml bovine serum albumin (BSA), $10 \mu \mathrm{M}$ lysophosphatidic acid (LPA), and various concentrations of recombinant human epidermal growth factor (rhEGF; 0.1-10 $\mu \mathrm{M}$ ). Detailed procedures for the stressed matrix contraction assay are described in Materials and methods. ${ }^{*} \mathrm{P}<0.05$ as indicated by the Student's t-test. 
those of the young fibroblasts, indicating that EGF may be an effective agent in enhancing the contractile activity of aged fibroblasts more effectively than that of young fibroblasts.

\section{Discussion}

EGF is one of various growth factors, and the mechanisms of action of EGF are linked with several intracellular signaling pathways involved with both motility and mitogenesis within interacting tissues. For these reasons, the biological effects of EGF have been studied in a wide range of human skin care applications, and EGF has been shown to accelerate wound healing and increase the rate of skin renewal in aging skin. However, the effects of EGF on the contractile capacity of aged human fibroblasts in a $3 \mathrm{D}$ collagen matrix have not yet been evaluated.

Current research is continuously developing the measurements of engineering dermal scaffolds that promote regeneration by incorporating growth factors into suitable scaffolds that mimic the cellular microenvironment (34). This approach relies upon a thorough understanding of the cell-ECM communications required to accomplish the physiological functions of the surrounding cells and tissue (35). Therefore, 3D collagen matrix systems may be a promising skin substitute for clinical applications.

Based on its similarity to human skin, in this study, we used the 3D collagen matrix system as an artificial skin model to assess the effects of rhEGF on 2 groups of fibroblasts representing young (12-year-old male) and aged (55-year-old male) fibroblasts, respectively. Specifically, we investigated the effects of rhEGF on the formation of clusters and contractility using SMC in a $3 \mathrm{D}$ collagen matrix system. As shown in Fig. 5, none of the treatments resulted in the decreased number of protrusions and dendritic extensions of young fibroblasts compared with aged fibroblasts. However, treatment with rhEGF promoted the contractile activity of the aged fibroblasts more effectively than that of the untreated fibroblasts (cultured under basal conditions). In addition, our results revealed that compared to culture under basal conditions, the contractile activity of the aged fibroblasts was significantly increased by culture with rhEGF in a SMC as with LPA treatment (Fig. 6), indicating that rhEGF may have a potential regenerative role in cellular function as a renewal agent.

Interactions between growth factors and cellular functions of aging cells are essential for the organization of the ECM-dependent processes, such as the organization of the collagen matrix, tissue development and wound healing. In addition, ECM proteins have also been shown facilitate the regeneration of skin (36). Experimental evidence has suggested that the expression of EGFR and other growth factor receptors is decreased in aging cells, and that this leads to diminished basal cell mitogenesis, motility and matrix formation (37). In cutaneous wound healing, EGF regulates fibroblast mobility and proliferation during all stages of wound healing, suggesting that it plays critical roles in coordinating wound repair through ECM remodeling $(37,38)$. Specifically, the loss of proliferative and migratory activity of aging fibroblasts coupled with the loss of wound closure ability and skin repair leads to a high risk of barrier disruption by surgical wound rupture and infection (17,39). Thus, the EGF-induced migra- tion and proliferation through the recovered levels of EGFR in aged fibroblasts has been suggested as a potential therapy for wound healing and skin recovery (40). In the present study, treatment with rhEGF was found to increase the proliferation of young fibroblasts compared with aged fibroblasts using cells in $2 \mathrm{D}$ culture (Fig. 3). We also investigated the effect of rhEGF on fibroblast migration activity using a 3D nested collagen matrix model. Our results revealed that rhEGF promoted the migration of both young and aged fibroblasts by stimulating dendritic extensions, and that PDGF, well known as a small $\mathrm{G}$ protein Rac-dependant promigratory activator $(32,41)$, increased the migration of both groups of cells, stimulating their protrusions (Fig. 4). Treatment with rhEGF affected fibroblast dendritic extensions, similar to treatment with PDGF. These results demonstrate that rhEGF exerts a promigratory effect on fibroblasts similar to PDGF, indicating that their promoting effects on migration may involve the same pathways.

Previous studies have demonstrated that age-related modifications are related to cell division, DNA synthesis and cell migration changed by mechanical forces $(33,42,43)$. It has been reported that fibroblast aging is associated with the disruption of cytoskeletal function, such as decreased actin polymerization, which in turn reduces cell elasticity $(36,44)$. Fibroblast functions such as cell motility and division regulated by the cytoskeleton have been investigated using 3D collagen lattices $(42,45)$. It has become increasingly clear over the years that the synthesis of ECM proteins is regulated by mechanical forces and that EGF can regulate these effects under both stressed and relaxed collagen gel conditions $(40,46,47)$. Fibroblast-mediated collagen matrices are a widely used in vitro model of tissue remodeling for examining different methods of restoring fibroblast functions (48-50). Indeed, the study of the activity of fibroblast migration and contraction using 3D collagen matrices has been regulated by serum and various factors, such as transforming growth factor (TGF)-1, PDGF and LPA (51). LPA is known as a small G protein Rho activator, and mediates the retraction of fibroblast dendritic extensions (52). LPA as a lipid agonist is a main factor of connective tissue remodeling stages that are related to the contractile activity of fibroblasts (31). Our results revealed that treatment with LPA stimulated fibroblast contractility to a greater extent than treatment with rhEGF; however, treatment with rhEGF enhanced the contractile activity of aged fibroblasts to a level similar or even higher than that of young fibroblasts (Fig. 6), indicating that rhEGF may be a more effective contractile factor of aged fibroblasts than LPA.

In conclusion, our results suggest that cell migration does not differ significantly between young and aged fibroblasts cultured with rhEGF on 3D collagen matrices, although rhEGF increased the migration of aged fibroblasts compared to no treatment (basal conditions). In addition, the contractile activities between the young and aged fibroblasts were quite different under basal conditions, although rhEGF enhanced the number of dendritic extensions in the aged fibroblasts compared with the young fibroblasts. These findings suggest that rhEGF is a potential renewal agent that acts to improve the contractile activity of aged fibroblasts through ECM regeneration in a $3 \mathrm{D}$ collagen matrix. Based on these data, we suggest that as rhEGF has promoting effects on the migration and 
contractility of aged fibroblasts, it may also have potential for use as a regenerative agent for aging skin.

\section{Acknowledgements}

The present study was supported by a grant from the Industrial Strategic Technology Development Program (10048038, Development of protein drug for hair follicle tissue formation using hUCB-MSC secreted proteins) funded by the Ministry of Trade, Industry and Energy (MOTIE, Korea) and Korea Evaluation Institute of Industrial Technology (KEIT).

\section{References}

1. Mukherjee PK, Maity N, Nema NK and Sarkar BK: Bioactive compounds from natural resources against skin aging. Phytomedicine 19: 64-73, 2011.

2. Iida I and Noro K: An analysis of the reduction of elasticity on the ageing of human skin and the recovering effect of a facial massage. Ergonomics 38: 1921-1931, 1995.

3. Seite S, Zucchi H, Septier D, Igondjo-Tchen S, Senni K and Godeau G: Elastin changes during chronological and photoageing: the important role of lysozyme. J Eur Acad Dermato Venereol 20: 980-987, 2006.

4. Enjoji M, Kotoh K, Iwamoto H, Nakamuta M and Nawata H: Selfregulation of type I collagen degradation by collagen-induced production of matrix metalloproteinase-1 on cholangiocarcinoma and hepatocellular carcinoma cells. In Vitro Cell Dev Biol Anim 36: 71-73, 2000.

5. Mecham RP, Broekelmann TJ, Fliszar CJ, Shapiro SD, Welgus HG and Senior RM: Elastin degradation by matrix metalloproteinases. Cleavage site specificity and mechanisms of elastolysis. J Biol Chem 272: 18071-18076, 1997.

6. Nanba D, Toki F, Barrandon Y and Higashiyama S: Recent advances in the epidermal growth factor receptor/ligand system biology on skin homeostasis and keratinocyte stem cell regulation. J Dermatol Sci 72: 81-86, 2013.

7. Danilenko DM, Ring BD and Pierce GF: Growth factors and cytokines in hair follicle development and cycling: recent insights from animal models and the potentials for clinical therapy. Mol Med Today 2: 460-467, 1996.

8. Marchese C, Chedid M, Dirsch OR, et al: Modulation of keratinocyte growth factor and its receptor in reepithelializing human skin. J Exp Med 182: 1369-1376, 1995.

9. Servold SA: Growth factor impact on wound healing. Clin Podiatr Med Surg 8: 937-953, 1991.

10. Brown GL, Curtsinger L III, Brightwell JR, et al: Enhancement of epidermal regeneration by biosynthetic epidermal growth factor. J Exp Med 163: 1319-1324, 1986.

11. Hollenberg MD and Cuatrecasas P: Epidermal growth factor: receptors in human fibroblasts and modulation of action by cholera toxin. Proc Natl Acad Sci USA 70: 2964-2968, 1973.

12. Cohen S and Taylor JM: Epidermal growth factor: chemical and biological characterization. Recent Prog Horm Res 30: 533-550, 1974.

13. Yarden Y and Shilo BZ: SnapShot: EGFR signaling pathway. Cell 131: 1018, 2007.

14. Akimoto N, Sato T, Sakiguchi T, Kitamura K, Kohno Y and Ito A: Cell proliferation and lipid formation in hamster sebaceous gland cells. Dermatology 204: 118-123, 2002.

15. Mascia F, Mariani V, Girolomoni G and Pastore S: Blockade of the EGF receptor induces a deranged chemokine expression in keratinocytes leading to enhanced skin inflammation. Am J Pathol 163: 303-312, 2003.

16. Schneider MR, Werner S, Paus R and Wolf E: Beyond wavy hairs: the epidermal growth factor receptor and its ligands in skin biology and pathology. Am J Pathol 173: 14-24, 2008.

17. Pastore S, Mascia F, Mariani V and Girolomoni G: The epidermal growth factor receptor system in skin repair and inflammation. J Invest Dermatol 128: 1365-1374, 2008.

18. Campbell D, Halligan S, Bartram CI, et al: Transvaginal power Doppler ultrasound in pelvic congestion. Acta Radiol 44: 269-274, 2003.

19. Gilchrest BA: Age-associated changes in the skin. J Am Geriatr Soc 30: 139-143, 1982.
20. Liu Y, Guyton KZ, Gorospe M, et al: Age-related decline in mitogen-activated protein kinase activity in epidermal growth factor-stimulated rat hepatocytes. J Biol Chem 271: 3604-3607, 1996.

21. Reenstra WR, Yaar M and Gilchrest BA: Aging affects epidermal growth factor receptor phosphorylation and traffic kinetics. Exp Cell Res 227: 252-255, 1996.

22. Freyman TM, Yannas IV, Pek YS, Yokoo R and Gibson LJ: Micromechanics of fibroblast contraction of a collagen-GAG matrix. Exp Cell Res 269: 140-153, 2001.

23. Naylor EC, Watson RE and Sherratt MJ: Molecular aspects of skin ageing. Maturitas 69: 249-256, 2011.

24. Yildirimer L, Thanh NT and Seifalian AM: Skin regeneration scaffolds: a multimodal bottom-up approach. Trends Biotechnol 30: 638-648, 2012.

25. Casasco A, Casasco M, Zerbinati N, Icaro Cornaglia A and Calligaro A: Cell proliferation and differentiation in a model of human skin equivalent. Anat Rec 264: 261-272, 2001.

26. Regnier M, Asselineau D and Lenoir MC: Human epidermis reconstructed on dermal substrates in vitro: an alternative to animals in skin pharmacology. Skin Pharmacol 3: 70-85, 1990.

27. Kim SY, Kim DS, Kwon SB, et al: Protective effects of EGCG on UVB-induced damage in living skin equivalents. Arch Pharm Res 28: 784-790, 2005.

28. Abe M, Ho CH, Kamm KE and Grinnell F: Different molecular motors mediate platelet-derived growth factor and lysophosphatidic acid-stimulated floating collagen matrix contraction. J Biol Chem 278: 47707-47712, 2003

29. Grinnell F, Rocha LB, Iucu C, Rhee S and Jiang H: Nested collagen matrices: a new model to study migration of human fibroblast populations in three dimensions. Exp Cell Res 312: 86-94, 2006.

30. Rheinwald JG and Green H: Serial cultivation of strains of human epidermal keratinocytes: the formation of keratinizing colonies from single cells. Cell 6: 331-343, 1975.

31. Jiang $\mathrm{H}$, Rhee $\mathrm{S}, \mathrm{Ho} \mathrm{CH}$ and Grinnell F: Distinguishing fibroblast promigratory and procontractile growth factor environments in 3-D collagen matrices. FASEB J 22: 2151-2160, 2008.

32. Seppa H, Grotendorst G, Seppa S, Schiffmann E and Martin GR: Platelet-derived growth factor in chemotactic for fibroblasts. J Cell Biol 92: 584-588, 1982.

33. Palmer HM, Higgins SP, Herring AJ and Kingston MA: Use of PCR in the diagnosis of early syphilis in the United Kingdom. Sex Transm Infect 79: 479-483, 2003.

34. Egana JT, Fierro FA, Kruger S, et al: Use of human mesenchymal cells to improve vascularization in a mouse model for scaffoldbased dermal regeneration. Tissue Eng Part A 15: 1191-1200, 2009.

35. Bianchi F, Rosi M, Vozzi G, Emanueli C, Madeddu P and Ahluwalia A: Microfabrication of fractal polymeric structures for capillary morphogenesis: applications in therapeutic angiogenesis and in the engineering of vascularized tissue. J Biomed Mater Res B Appl Biomater 81: 462-468, 2007.

36. Delvoye P, Wiliquet P, Leveque JL, Nusgens BV and Lapiere CM: Measurement of mechanical forces generated by skin fibroblasts embedded in a three-dimensional collagen gel. J Invest Dermatol 97: 898-902, 1991 .

37. Ashcroft GS, Horan MA and Ferguson MW: The effects of ageing on cutaneous wound healing in mammals. J Anat 187: $1-26,1995$.

38. Lawrence WT and Diegelmann RF: Growth factors in wound healing. Clin Dermatol 12: 157-169, 1994.

39. Gerber PA, Enderlein E and Homey B: The Koebner-phenomenon in epidermal growth factor receptor inhibitor-induced cutaneous adverse effects. J Clin Oncol 26: 2790-2792, 2008.

40. Shiraha H, Gupta K, Drabik K and Wells A: Aging fibroblasts present reduced epidermal growth factor (EGF) responsiveness due to preferential loss of EGF receptors. J Biol Chem 275: 19343-19351, 2000.

41. Anand-Apte B, Zetter BR, Viswanathan A, et al: Platelet-derived growth factor and fibronectin-stimulated migration are differentially regulated by the Rac and extracellular signal-regulated kinase pathways. J Biol Chem 272: 30688-30692, 1997.

42. Takeda K, Gosiewska A and Peterkofsky B: Similar, but not identical, modulation of expression of extracellular matrix components during in vitro and in vivo aging of human skin fibroblasts. J Cell Physiol 153: 450-459, 1992.

43. Reed MJ, Ferara NS and Vernon RB: Impaired migration, integrin function, and actin cytoskeletal organization in dermal fibroblasts from a subset of aged human donors. Mech Ageing Dev 122: 1203-1220, 2001. 
44. Rao KM and Cohen HJ: Actin cytoskeletal network in aging and cancer. Mutat Res 256: 139-148, 1991.

45. Rattan SI: Theories of biological aging: genes, proteins, and free radicals. Free Radic Res 40: 1230-1238, 2006.

46. Teti A: Regulation of cellular functions by extracellular matrix. J Am Soc Nephrol 2: S83-S87, 1992.

47. Werner S, Krieg T and Smola H: Keratinocyte-fibroblast interactions in wound healing. J Invest Dermatol 127: 998-1008, 2007.

48. Janmey PA and Weitz DA: Dealing with mechanics: mechanisms of force transduction in cells. Trends Biochem Sci 29: 364-370, 2004.

49. Guck J, Schinkinger S, Lincoln B, et al: Optical deformability as an inherent cell marker for testing malignant transformation and metastatic competence. Biophysical J 88: 3689-3698, 2005.
50. Princz MA and Sheardown H: Modified dendrimer cross-linked collagen-based matrices. J Biomater Sci Polym Ed 23: 2207-2222, 2011.

51. Ridley AJ, Paterson HF, Johnston CL, Diekmann D and Hall A: The small GTP-binding protein rac regulates growth factorinduced membrane ruffling. Cell 70: 401-410, 1992.

52. Yee HF Jr, Melton AC and Tran BN: RhoA/rho-associated kinase mediates fibroblast contractile force generation. Biochem Biophys Res Commun 280: 1340-1345, 2001. 